\title{
Estimation of Power Grid Topology Parameters through Pilot Signals
}

\author{
S. Neshvad ${ }^{1}$, H. Margossian ${ }^{1}$ and J. Sachau ${ }^{1}$ \\ ${ }^{1}$ Interdisciplinary Centre for Security, Reliability and Trust (SnT), \\ University of Luxembourg \\ 4, rue Alphonse Weicker, L-2721 Luxembourg \\ e-mail: surena.neshvad@uni.lu, harag.margossian@uni.lu, jürgen.sachau@uni.lu
}

\begin{abstract}
The distribution grid has been the source of an increased amount of electricity production in recent years. Coupled with the adoption of open energy markets, this event has significantly complicated the powerflows on the distribution grid, urging network operators to invest in advanced control and monitoring tools in order to optimize the efficiency and reliability of the system. In this context, a method to estimate the connection status of distributed generators and the system topology is proposed in this paper, the goal being to obtain up to date information on the power system network's configuration. This will enable grid operators to have visibility on the status of the distribution grid and react proactively to problematic situations that might arise. The proposed topology estimation method relies on injected pilot signals through generators feeding in power. Pilot voltage stimulations are injected from distributed generators and the induced currents effects are measured at several nodes in the system. The measured data is evaluated through correlation, and a weighed least-square algorithm, applied to the network's dynamic model, estimates those unknown parameters and provides an accurate snapshot of the power network topology. The performance of the proposed method is evaluated on a small scale distribution network.
\end{abstract}

\section{Key words}

Power Network Topology Identification, System Parameter Estimation, Weighed Least Squares, Active Identification

\section{Introduction}

Measurement and network parameter errors have a negative impact on the data needed by the power system operator for operation and control. Such errors can be due to transmission line parameters, circuit breaker status or Distributed Generator (DG) connection status [1]. State Estimation (SE) and other studied control mechanisms such as network topology reconfiguration [2], adaptive protection and short circuit calculation tools [3] use this model in order to optimize powerflows and guarantee proper behavior of protective devices.

State Estimation is performed under the assumption that network topology is fixed and assessed correctly. However, the power network configuration can change for numerous reasons. Also, other parameters such as transmission lines impedances and transformer tap position can vary over time as well. The busbar connections can also be modified by changing the status of the circuit breakers on the transmission lines. In recent years, with the addition of large amount of distributed energy sources on the power grid, the concept of intentionally modifying the network's topology has been studied [2]. Several topology estimation solutions have been proposed, and they are mostly based on alterations of existing state estimation. Some methods analyze the residual of state estimation and identify correlation patterns linking residuals to topology configurations [4-5]. This analysis is performed in parallel with state estimation, and is added as an extra step to the algorithm. Other methods apply an extended state vector and use constraints on the system parameters in order to estimate the unknown variables [6-7].

In this paper, topology estimation is performed based on active identification [8]. The voltage is distorted at the DG by injecting a broad frequency pilot signal, and the resulting current distortions are processed through correlation with the injected voltage signals. Active identification of the impedance at the inverter's Point of Common Coupling (PCC) for detecting islanding conditions has been researched in numerous studies [910]. In this research the harmonic rich currents generated at the inverter are additionally evaluated at neighboring nodes in the power network. Based on the research in [11], Pseudo Random Binary Sequences (PRBS) signals are used to create a broad-spectrum stimulation. Pilot signals are sent from several generators in the power system and the induced currents are measured at several locations. All measurement results are then combined in equations using the network's admittance matrix. Finally a Weighed Least Square (WLS) algorithm is applied in order to infer the unknown system variables.

The proposed active identification method using PRBS stimulation signals is covered in Section 2. A system model is presented, and a characterization method using several measurements is described. In Section 3, a WLS algorithm is applied in order to extract the unknown parameters of the system's admittance matrix. Finally, simulation results for a test system are provided in Section 4 , and an analysis of system performance and result accuracy is provided. 


\section{Topology Identification through Active Identification}

\subsection{Pilot Signal Properties}

Pseudo-Random Binary Sequences are binary streams that are deterministic but 'pseudo-random' over their run length. They have been used as pilot signals for system characterization in many engineering fields [12]. For Maximum Length Sequences (MLS), for a given seed, a polynomial of length $L$ will generate a deterministic sequence of ' 1 's and ' 0 's of $2^{L}-1$ elements [16]. Depending on the relationship between the code-length, sampling frequency and code frequency, the PRBS exhibits a 'white noise-like' spectrum for a defined frequency range, with zeros occurring at multiples of the PRBS clock sampling frequency. The code length and its sampling frequency limit the resolution of the spectrum that will be stimulated. The logic required for PRBS implementation consists of shifters and XORs and can easily be implemented in software. Another advantage of PRBS is that even very low amplitude pilot signals can produce accurate estimation results, since their effect is aggregated over a complete run length.

The PRBS signal can be detected in a high interference environment by a correlator who is aware of its sequence. The tasks depicted in Figure 1 summarize the operations to be performed for impulse response determination.

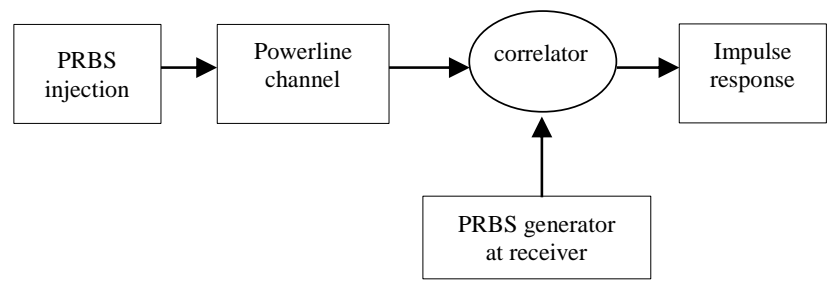

Figure 1: Impulse response determination setup

By applying a Fourier transform to the obtained impulse response, the transfer function of the system in frequency domain is established.

\subsection{Power System Model under Study}

The distribution network topology depicted in Figure 2 is considered for the application of the proposed identification algorithm. It consists of a power generator on a high voltage network connected to a voltage transformer distributing power through two feeders. The feeders are branched twice to distribute power to the loads. The network comprises three switches for topology reconfiguration, seven nodes, and six transmission lines. The complete network can be fed through feeder 1 , feeder 2 or both depending on the status of the switches $S 1, S 2$ and $S 3$. Additionally a meshed network can be produced if all switches are connected. DG1 and DG3 are monitoring DGs and periodically inject PRBS sequences on the grid. The connection status of DG5 and DG6 is intermittent and not known by the operator. The objective of the topology identification algorithm is to evaluate the status of the switches $S 1, S 2$ and $S 3$ as well as the connection status of $D G 5$ and $D G 6$, represented by the switches $D 1$ and $D 2$. The transmission lines are modelled as resistive-inductive components, which is accurate enough to model the dynamics of the transmission lines for the considered frequency ranges and cable lengths.

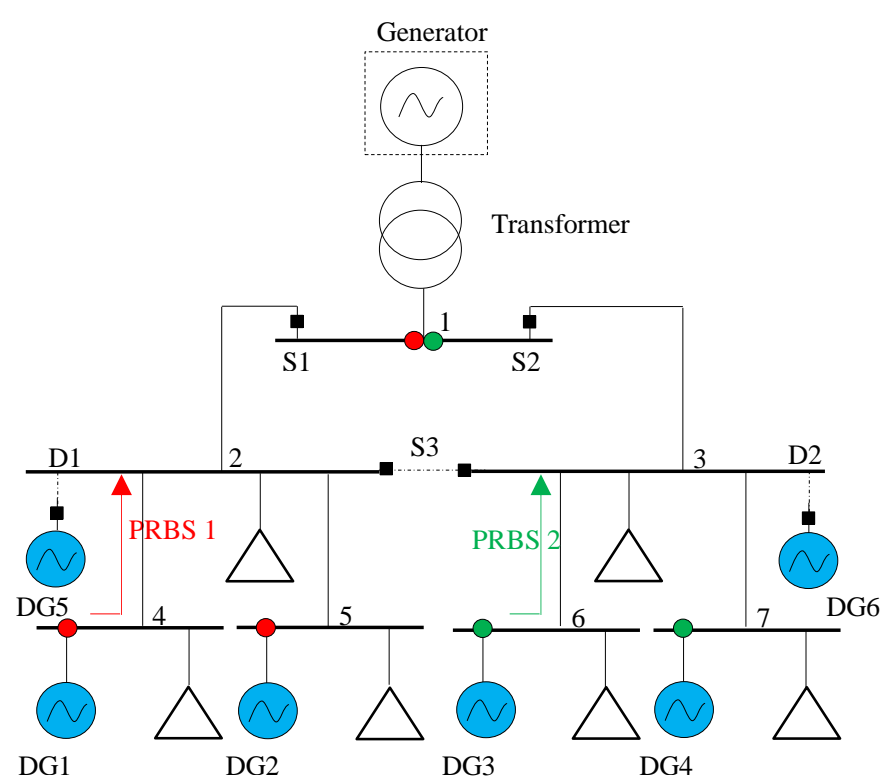

Figure 2: Distribution network model used for system identification

For typical residential loads, the relative high equivalent impedance has a minimal impact on harmonic current flows and ignoring it doesn't modify the estimations considerably. The equivalent impedance of the MV transformer and upstream grid is chosen according to [13], a resistive-inductive model is used for the substation transformer. The electrical parameters of the system components are described in Table 1 in Section IV.

\subsection{Active Identification}

PRBS patterns are injected from $D G 1$ and $D G 3$, and the resulting current patterns are measured at various locations in the network, marked with a red dot for $D G 1$ and green dot for DG3 in Figure 2. Previous research in [14] has shown that the presence of the PRBS can be detected through continuous correlation with the PRBS code and a correlation peak locates the instant and the duration of the received pattern. Subsequently, the transfer function relating the PRBS voltage patterns to the measured current patterns can be established. Equation (1) shows the results of this operation for the described model, $h(n)$ being the transfer function of the propagation path and $R_{s r}$ the autocorrelation of the stimulation:

$$
\begin{aligned}
v_{S}(t) * i_{r}(t) & =\sum_{n=s t a r t_{-} p r b s_{-} i d x}^{\text {end_prbs_idx }} v_{S}(n) i_{r}(n+m) \\
& =\sum_{n=\text { start_prbs_idx }}^{\text {end_prbs_idx }} h(n) R_{s r}(m-n)
\end{aligned}
$$

Transposed to the frequency domain, equations (2-3) are obtained:

$$
\bar{V}_{s} \cdot I_{r}(\omega)=H_{i}(\omega)\left|V_{s}(\omega)\right|^{2}
$$




$$
H_{i}(\omega)=\frac{\bar{V}_{s} \cdot I_{r}(\omega)}{\left|V_{s}(\omega)\right|^{2}}
$$

Equation (3) indicates the dynamic relationship between the sender voltage and receiver current. It represents the spectral dependency between the injected voltage harmonics with the resulting current harmonics at the receiver.

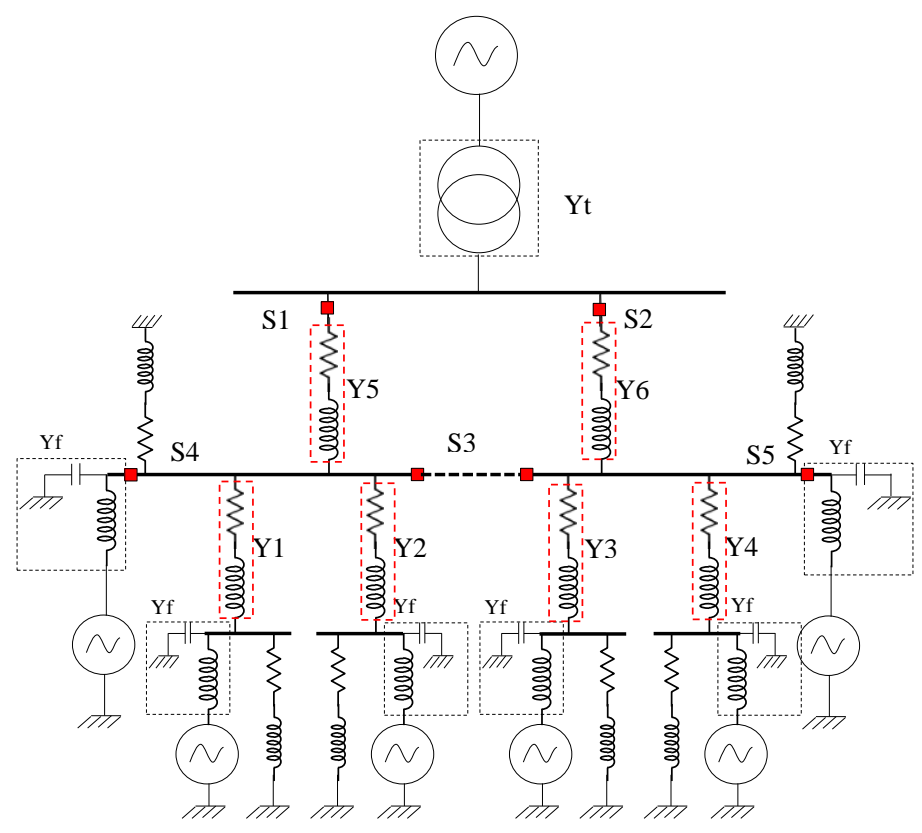

Figure 3: Electrical representation of distribution network model

The electrical model of the system under study is depicted in Figure 3. The output filters of all DGs are LC filters and their admittances $Y f$ are assumed identical. The unknown parameters in the system are switch settings $S 1$ $S 3$ and DG connection status D1-D2. These are binary unknowns, and their value represents the connection status of the switch/DG. When the switch $S 1$ is open, the parameter $S 1$ on the Ymatrix, has the value 0, which represents an open connection between node 1 and node 2 . For a closed connection, $S 1$ takes the value 1 , and the admittance of the line will be the parameter used in the admittance matrix.

The line impedances $Y 1-Y 6$, consist of a resistive and an inductive component. The admittance matrix of the network is represented by equations (4-11).

$$
\begin{gathered}
\begin{array}{c}
Y \text { matrix } \\
=\left[\begin{array}{ccccccc}
Y_{\text {sum } 1} & -S 1 \cdot Y 5 & -S 2 \cdot Y 6 & 0 & 0 & 0 & 0 \\
-S 1 \cdot Y 5 & Y_{\text {sum } 2} & -S 3 \cdot Y s 3 & -Y 1 & -Y 2 & 0 & 0 \\
-S 2 \cdot Y 6 & -S 3 \cdot Y s 3 & Y_{\text {sum } 3} & 0 & 0 & -Y 3 & -Y 4 \\
0 & -Y 1 & 0 & Y_{\text {sum } 4} & 0 & 0 & 0 \\
0 & -Y 2 & 0 & 0 & Y_{\text {sum } 5} & 0 & 0 \\
0 & 0 & -Y 3 & 0 & 0 & Y_{\text {sum } 6} & 0 \\
0 & 0 & -Y 4 & 0 & 0 & 0 & Y_{\text {sum } 7}
\end{array}\right] \\
Y_{\text {sum } 1}=Y_{t}+Y 5 \cdot S 1+Y 6 \cdot S 2 \\
Y_{\text {sum } 2}=Y 5 \cdot S 1+Y 1+Y 2+Y s 3 \cdot S 3+Y f \cdot D 1
\end{array} \\
Y_{\text {sum } 3}=Y 6 \cdot S 2+Y 3+Y 4+Y s 3 \cdot S 3+Y f \cdot D 2
\end{gathered}
$$

$$
\begin{gathered}
Y_{\text {sum } 5}=Y 2+Y f \\
Y_{\text {sum } 6}=Y 3+Y f \\
Y_{\text {sum } 7}=Y 4+Y f
\end{gathered}
$$

$D G 1$ and $D G 3$ periodically inject PRBS patterns on the power network. The injections are orthogonal [12], thus their interference will be minimal if concurrent injection from two DGs happens. At DG1-DG4 and the transformer substation, a receiver correlates the incoming current with the PRBS codes of the senders. Upon arrival of the stimulation, a correlation peak is detected [14]. Using equations (3), the transfer function between the emitter and the receiver can be established.

The cross-correlation of PRBS with uncorrelated signals is minimal, thus for the elaboration of the system equations to be solved it will assumed that the PRBS is the only stimulation present for the considered frequencies during its injection. For the seven node system depicted in Figure 2, the relationship between Vsender and Ireceiver can be expressed based on standard methods [15]. For instance, Vsnd4 represents the voltage spectrum created at node 4, and $I r C v_{1-4}$ are the current spectrum measured at node 1 due to Vsnd4. Vsnd4 is the pattern injected at the $D G l$, and is known in advance for each monitoring DG. Ircv4 is detected, measured and processed at the receiver when the PRBS pattern is incoming. Transposing Vsnd4 and Ircv4 to the Fourier domain, we obtain for each frequency a set of equations relating those measurements at nodes 1,4 , and 5 , by linking them to the unknown parameters of the admittance matrix. The same operation can be performed with $D G 3$ and a total set of 6 equations can be established at each frequency. The system to be solved is shown in matrix (12): $\left[\begin{array}{cccc}0 & 0 & 0 & 0 \\ 0 & 0 & 0 & 0 \\ 0 & 0 & 0 & 0 \\ \text { Vsnd4 } & 0 & 0 & 0 \\ 0 & 0 & 0 & 0 \\ 0 & 0 & \text { Vsnd6 } & 0 \\ 0 & 0 & 0 & 0\end{array}\right]=$ Ymatrix $^{-1} \cdot\left[\begin{array}{cccc}\operatorname{Ircv_{1-4}} & 0 & \operatorname{Ircv}_{1-6} & 0 \\ 0 & 0 & 0 & 0 \\ 0 & 0 & 0 & 0 \\ I r c v_{4-4} & 0 & 0 & 0 \\ I r c v_{5-4} & 0 & 0 & 0 \\ 0 & 0 & \operatorname{Ircv}_{6-6} & 0 \\ 0 & 0 & \operatorname{Ircv}_{7-6} & 0\end{array}\right]$

The equations linking measurements to unknown variables have established and in the next section, a Weighed-Least-Squares method will be applied to estimate the unknown parameters in the system.

\section{Solving the System Equation using WLS}

\subsection{Overview of WLS}

A measurement model to be solved can be written as:

$$
z=h\left(t_{p}, c_{p}\right)+e
$$

where $z$ is the measurement vector, $h\left(t_{p}, c_{p}\right)$ is the nonlinear function relating the measurements to the system parameters [16], consisting in our case of topology and connection parameters. $t_{p}$ is the vector containing network topology and connection parameters, $c_{p}$ is the 
vector of DG connection parameters, $e$ is the vector of measurement errors.

There are $m$ measurements and $n$ variables to be determined, with the over-specification constraint that $n<$ $m$. The WLS parameter estimation can be formulated mathematically as an optimization problem with a quadratic objective function with additional equality constraints implemented as pseudo-measurements, the residual being defined as:

$$
r=z-h(x, p)
$$

The goal is to minimize the objective function:

$$
\begin{aligned}
J(x, p) & =\sum_{i=1}^{m} \frac{\left(z_{i}-h_{i}(x)\right)^{2}}{R_{i i}} \\
& =[z-h(x, p)]^{T} R^{-1}[z-h(x, p)]
\end{aligned}
$$

where $R$ is the covariance matrix related to the estimated accuracy of each measurement. The minimum of the objective function can be obtained if:

$$
g(x)=\frac{d J(x)}{d x}=-H^{T}(x) R^{-1}[z-h(x)]=0
$$

$H(x)$ being the Jacobian of matrix $h(x)$

$$
H(x)=\left\lceil\frac{d h}{d x}\right]
$$

Expanding $g(x)$ into its non-linear Taylor series, we obtain:

$$
g(x)=g\left(x^{k}\right)+G\left(x^{k}\right)\left(x-x^{k}\right)+\cdots=0
$$

Neglecting the higher order terms leads to an iterative solution scheme known as the Gauss-Newton method as shown below:

$$
x^{k+1}=x^{k}-\left[G\left(x^{k}\right)\right]^{-1} \cdot g\left(x^{k}\right)
$$

where $k$ is the iteration index, $x^{k}$ is the solution vector at iteration $k$

$$
\begin{gathered}
G(x)=\frac{d g\left(x^{k}\right)}{d x}=H^{T}\left(x^{k}\right) \cdot R^{-1} \cdot H\left(x^{k}\right) \\
g\left(x^{k}\right)=-H^{T}\left(x^{k}\right) \cdot R^{-1} \cdot\left(z-h\left(x^{k}\right)\right)
\end{gathered}
$$

The iterative process is usually stopped once convergence is assumed, that is for a threshold $\epsilon$, once

$$
\left|\Delta x^{k}\right| \leq \epsilon
$$

\subsection{WLS algorithm on model under study}

The described WLS algorithm will be adapted to the system model. The measured parameter is the transfer function of the system $H(\omega)$, which corresponds to the relationship between the stimulation at the source and the current harmonics at the receiver. The function relating measurements to system parameters is the modified admittance matrix (4) which contains information on the Switch settings $t_{p}$ and DG connection status $c_{p}$.

$$
h\left(t_{p}, c_{p}\right)=\operatorname{Ymatrix}\left(t_{p}, c_{p}\right)
$$

These parameters are estimated at first, and the estimation is adjusted iteratively. The Jacobian of the inverse of the admittance matrix is computed for each parameter and the iterative process modifies the unknown variables in order to minimize the residual. Thus, the objective function is minimized according to the equations (14-22). The weight (inverse of the covariance) for each measurement is set based on the reliability of the measurement, which in turn depends on its Signal-toNoise Ratio (SNR). The value for $R_{i i}$, corresponding to weight, can be dynamically computed for each measurement [14]. The WLS algorithm is repeated for a set of frequencies, in order to provide additional data points for confirming the switch settings.

In the studied system, there are two PRBS injectors, acting as senders, and five receivers, detecting and measuring each of the current stimulations produced by these senders. There are 5 unknowns for the switch and DG connection settings, and 6 equations linking those unknowns for each frequency, as depicted in (12).

The model and the proposed algorithm are run on Simulink \& Matlab is Section 4, and an analysis of the outcome and performance provided.

\section{Simulations}

Simulations are based on the system described in Section II and Section III. The model is based on Simulink/Matlab for the PRBS injection/measurement and WLS algorithm. The characteristics of the system to be estimated, including stimulation parameters and receiver settings are listed in Table I. The parameters to be estimated are marked in italics. The network considered is the one depicted in Figure 2.

Table I. - Simulation Settings

\begin{tabular}{|l|l|}
\hline Stimulation parameters & \\
\hline PRBS code length & 4093 \\
\hline PRBS 1 & $x^{12}+x^{8}+x^{2}+x+1$ \\
\hline PRBS 2 & $x^{12}+x^{6}+x^{4}+x+1$ \\
\hline PRBS 3 & $x^{12}+x^{6}+x^{5}+x^{3}+1$ \\
\hline PRBS 4 & $50 \mathrm{KHz}$ \\
\hline Sampling frequency & $12800 \mathrm{~Hz}$ \\
\hline Carrier frequency & 256 \\
\hline $\begin{array}{l}\text { Codes per fundamental } \\
\text { cycle }\end{array}$ & \\
\hline Electrical system params & $0.1 \Omega, 2 \mathrm{mH}$ \\
\hline Line 1 impedance Zl1 & $0.3 \Omega, 6 \mathrm{mH}$ \\
\hline Line 2 impedance Zl2 & $0.2 \Omega, 1 \mathrm{mH}$ \\
\hline Line 3 impedance Zl3 & $0.1 \Omega, 2 \mathrm{mH}$ \\
\hline Line 4 impedance Zl4 & $0.3 \Omega, 3 \mathrm{mH}$ \\
\hline Line 5 impedance Zl5 & $0.1 \Omega, 1 \mathrm{mH}$ \\
\hline Line 6 impedance Zl6 & $5 \Omega, 9.45 \mathrm{e} 1 \mu \mathrm{F}-10 \mathrm{mH}$ \\
\hline $\begin{array}{l}\text { Inverter Output filter } \mathrm{Zc}- \\
\text { Zf }\end{array}$ & \\
\hline Transformer Impedance Zt & $0.12 \Omega, 20 \mathrm{mH}$ \\
\hline Unknown parameters & $S W 1=1, S W 2=0, S W 3=1$ \\
\hline Switch settings & $D G 1=0, D G 2=1$ \\
\hline DG connection setting & \\
\hline
\end{tabular}


Line parameters are based on the power network in Luxembourg operated by Creos. The first step of the algorithm is the transfer function estimation following the PRBS injections, based on equation (3). The measurement locations in the grid are marked in red/green in Figure 2.

Figure 4 shows the results of transfer function identification from source $D G 1$ (node 4) and DG3 (node 6). The straight line corresponds to the theoretical value to be obtained based on the calculated value of the equivalent model. The noisy/colored lines are the results obtained through PRBS injection and correlation as described in equation (3). As stated in Section III, the weights associated to these noisier measurements will be lower in the WLS algorithm. The measurements close to the PRBS source will have higher impact during WLS, while measurements electrically far from the source provide low reliability data, associated with a small weight.
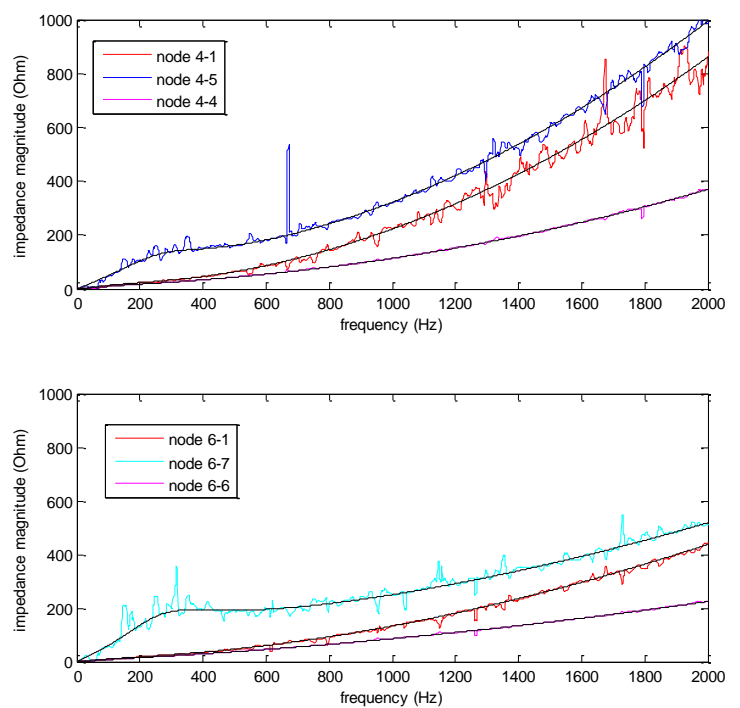

Figure 4: Theoretical and estimated transfer function from source nodes to destination nodes

Data is collected and processed for both PRBS sources, then evaluated. Once the transfer functions are established, they will be used for estimating the unknowns of the system. The data from the simulation is processed through WLS, using the Ymatrix and the equations elaborated in Section II and III. The results of each of the WLS runs is listed and analyzed In Table II. In the test settings, the switch settings are set to ' 1 ' ' 0 ' ' 1 ', and the DG settings to ' 0 ' ' 1 ': the complete network is powered by the left feeder, and the DG on node 2 is disconnected. For the initial 'guess' of the WLS algorithm, all parameters are set to 0 . The algorithm is run for the frequency range $80-2000 \mathrm{~Hz}$, with $100 \mathrm{~Hz}$ intervals. The results obtained through this procedure are shown in Table II. The weighted average for each of the estimations at the bottom of the table corresponds to the result for each frequency, compounded by a factor inversely proportional to the residue of the WLS run. Since, badly converging runs usually end with higher residues, their impact on the final results is reduced without additional steps of data filtering and processing.
Table II. - Topology Estimation Results

\begin{tabular}{|c|c|c|c|c|c|c|}
\hline & S1 & S2 & S3 & D1 & D2 & \\
\hline & \multicolumn{5}{|c|}{ Real Topology values to be found } & \\
\hline & 1 & 0 & 1 & $\mathbf{0}$ & 1 & \\
\hline & \multicolumn{5}{|c|}{ Initial WLS parameter setting } & \\
\hline & $\mathbf{0}$ & $\mathbf{0}$ & $\mathbf{0}$ & $\mathbf{0}$ & $\mathbf{0}$ & \\
\hline Frequency & \multicolumn{5}{|c|}{ WLS result } & residue \\
\hline 80 & 0.99 & 0.01 & 0.97 & 0.01 & 0.86 & 0.16 \\
\hline 180 & 0.99 & 0.03 & 0.57 & 0.08 & 0.91 & 0.58 \\
\hline 280 & 0.99 & 0.01 & 0.99 & 0.01 & 0.94 & 0.30 \\
\hline 380 & 0.78 & 0.01 & 0.99 & 0.01 & 0.99 & 0.24 \\
\hline 480 & 0.88 & 0.01 & 0.99 & 0.09 & 0.99 & 0.65 \\
\hline 580 & 0.99 & 0.01 & 0.99 & 0.01 & 0.99 & 0.32 \\
\hline 680 & 0.75 & 0.01 & 0.88 & 0.01 & 0.99 & 0.64 \\
\hline 780 & 0.79 & 0.15 & 0.99 & 0.09 & 0.91 & 1.02 \\
\hline 880 & 0.99 & 0.01 & 0.99 & 0.01 & 0.70 & 0.87 \\
\hline 980 & 0.89 & 0.01 & 0.78 & 0.03 & 0.94 & 1.00 \\
\hline 1080 & 0.88 & 0.01 & 0.94 & 0.88 & 0.05 & 4.10 \\
\hline 1180 & 0.84 & 0.01 & 0.91 & 0.08 & 0.91 & 1.36 \\
\hline 1280 & $\begin{array}{l}0.75 \\
\end{array}$ & $\begin{array}{l}0.01 \\
\end{array}$ & 0.89 & 0.11 & 0.94 & 0.73 \\
\hline 1380 & 0.99 & 0.01 & 0.99 & 0.01 & 0.99 & 0.07 \\
\hline 1480 & 0.69 & 0.01 & 0.93 & 0.01 & 0.99 & 0.29 \\
\hline 1580 & 0.81 & $\begin{array}{l}0.01 \\
\end{array}$ & 0.95 & 0.10 & 0.98 & 0.94 \\
\hline 1680 & $\begin{array}{l}0.69 \\
\end{array}$ & 0.01 & 0.99 & 0.21 & $\begin{array}{l}0.99 \\
\end{array}$ & 1.92 \\
\hline 1780 & 0.86 & 0.10 & 0.78 & 0.99 & 0.01 & 5.94 \\
\hline 1880 & 0.79 & 0.01 & 0.86 & 0.23 & 0.67 & 1.64 \\
\hline 1980 & 0.84 & 0.11 & 0.93 & 0.01 & $\begin{array}{l}0.99 \\
\end{array}$ & 1.58 \\
\hline $\begin{array}{l}\text { Weighted } \\
\text { average }\end{array}$ & 0.94 & 0.03 & 0.96 & 0.08 & 0.91 & \\
\hline
\end{tabular}

The results of the final weighted average show that the switch settings are estimated correctly, assuming that the final result will be rounded up/down. Simulations for other switch settings have also provided accurate estimations, without addition of other data analysis or heuristics to improve convergence accuracy. For some frequencies, such as $1080 \mathrm{~Hz}$, the WLS algorithm has converged to the wrong value. This can be attributed to the highly non-linear nature of the system, since a parameter jump in the iterative procedure can lead the parameter to an erroneous convergence. Nevertheless this error can be tolerated, since the majority of the frequencies converge correctly and since the bad results typically end the WLS run with higher residues, making their contribution to the weighted average less prevalent.

\section{Conclusions}

The extensive addition of DG to the distribution network is making an active monitoring and control of its operations increasingly necessary. We have proposed in this research a method for estimating the topology of the power network using pilot signals. We inject predefined coded signals from selected DGs, and measure the current response of these signals at several nodes on the distribution network. The combination of these measurements allows us to obtain a good estimate for the unknown switch and DG connection parameters. The proposed method can be applied to any other unknown parameters of the system, as long as its effect on the stimulations can be assessed in the admittance matrix. Thus estimation of line parameters, variable large loads or transformer tap settings can be contemplated and will be addressed in further research.

The tool described in this research would be a valuable asset to enable utility providers to modernize the complex distribution network, without the addition of extensive communication infrastructure. It provides a novel monitoring solution that is complementary to traditional EMS tools. 


\section{Acknowledgment}

This work was conducted at the "Interdisciplinary Centre for Security, Reliability and Trust" (SnT) at the University of Luxembourg in collaboration with CREOS S.A., the Luxemburgish utility provider. It is supported by the National Research Fund, Luxembourg.

\section{References}

[1] O. Alsac, N. Vempati, B. Slott, A. Monticelli, "Generalized State Estimation", 20th International Conference on Power Industry Applications 1997

[2] I. Bilibin, F. Capitanescu, J. Sachau, "Overloads Management in Active Radial Distribution Systems : an Optimization Approach Including Network Switching", IEEE 5th International Symposium on Power Electronics for Distributed Generation Systems (PEDG)

[3] H. Margossian, J. Sachau, "Short Circuit Calculation in Networks with a High Share of Inverter Based Distributed Generation"

[4] W. Liu, F. Wu, and S. Lun, "Estimation of parameter errors from measurement residuals in state estimation," IEEE Trans. Power Syst., vol. 7, no. 1, pp. 81-89, Feb. 1992.

[5] T. Van Cutsem and V. Quintana, "Network parameter estimation using online data with application to transformer tap position estimation," Proc. Inst. Elect. Eng., vol. 135, pp. 31-40, Jan. 1988.

[6] E. Handschin and E. Kliokys, "Transformer tap position estimation an bad data detection using dynamic signal modeling,” IEEE Trans. Powe Syst., vol. 10, no. 2, pp. 810 817, May 1995.

[7] I. Slutsker and S. Mokhtari, "Comprehensive estimation in power systems: State, topology and parameter estimation," in Proc. American Power Conf., Chicago, IL, Apr. 1995, Paper 170.

[8] L. Ljung, T. Glad, "Modeling of Dynamic Systems", Prentice Hall 1994

[9] L. Asiminoaei, E. Teodorescu, F. Blaabjerg, U. Borup, "Implementation and Test of an Online Embedded Grid Impedance Estimation Technique for PV Inverters, IEEE Trans. on Ind. Electronics, Aug 2005

[10] H. Langkowski, T Thanh, K. Dettmann, D. Schulz, „Grid Impedance Determination - Relevancy for Grid Integration of Renewable Energy Systems", IEEE 35th Annual Industrial Electronics'09, November 2009

[11] S. Neshvad, S. Chatzinotas, J. Sachau, "Online Determination of Grid Impedance Spectrum through Pseudo-Random Excitation of a Pulse Width Modulator", ICREPQ, April 2014

[12] R.N. Mutagi, "Pseudo Noise Sequences for Engineers", IEEE Electronics and Communication Engineering Journal, April 1996

[13] $\mathrm{ABB}$, "MV/LV transformer substations: theory and examples of short-circuit calculation", Technical Application Papers, February 2008

[14] S. Neshvad, S. Chatzinotas, J. Sachau, Wideband Identification of Power Network Parameters Using PseudoRandom Binary Sequences on Power Inverters", IEEE Transactions on Smart Grid 2015

[15] A. Monticelli, "Electric Power System State Estimation", Proceedings of the IEEE, February 2000

[16] A. Abur, A.G. Exposito, "Power System State Estimation, Theory and Implementation", CRC Press ISBN 0-8247$5570-7$ 